\title{
Primary microcephaly-mild intellectual disability-young-onset diabetes syndrome
}

INSERM

\section{Source}

INSERM. (1999). Orphanet: an online rare disease and orphan drug data base. Primary microcephaly-mild intellectual disability-young-onset diabetes syndrome. ORPHA:391408

Primary microcephaly-mild intellectual disability-young-onset diabetes syndrome is a rare, genetic, syndromic intellectual disability disorder characterized by congenital, persistent microcephaly, low birth weight, short stature, childhood-onset seizures, global development delay, mild intellectual disability, and adolescent or young adult-onset diabetes mellitus. Gait ataxia, skeletal abnormalities, dorsocervical fat pad, and infantile cirrhosis may also be associated. Brain morphology is typically normal, although delayed myelination and hypoplastic brainstem have been reported. 